\title{
Social supports among college students and measures of alcohol use, perceived stress, satisfaction with life, emotional intelligence and coping
}

\author{
Carolyn Whitney \\ Assistant Professor of Psychology \\ St Michael's College \\ Colchester, Vermont, USA
}

\begin{abstract}
In this study I examined three domains of social supports among college students (close friends, casual friends and safe adults to turn to) in relation to indices of wellbeing and coping. Measures of positive wellbeing were most strongly associated with the safe adults domain of social support followed by the close friends domain of social support. Casual friends were associated only with measures of problem alcohol consumption but not with indices of wellbeing. Students with five or more safe adults to turn to as compared to four or fewer reported significantly lower perceived stress, greater satisfaction with life, higher emotional intelligence, better academic performance and lower problem drinking scores. The domain of safe adults was associated with the largest array of wellbeing indices of all three social support domains. Future research should examine additional measures of wellbeing that may be associated with distinct domains of support.
\end{abstract}

\section{Introduction}

The transition to life in college can be a challenging time for many young adults, thus perceptions of social support and being cared for by those in one's life can be very important to levels of wellbeing. Among high school students, Chou (2000) found that family social support was associated with lower levels of depression while friend social support was associated with lower levels of anxiety. Among college students, Clara et al. (2003) found that both family and friend social supports were associated with lower levels of depression, while Davis, Morris and Kraus (1998) found social support from friends to be the most powerful support associated with college student wellbeing followed by that of parents and romantic partners. 
Whether actual or perceived, social support reflects overall feelings that one is cared for, accepted and that in difficult times one will have others to turn to who will provide assistance and help (Sarason, Sarason and Pierce 1990; Davis, Morris and Kraus 1998). Clara et al. suggest that it is the perception of global social support that appears to provide a buffering effect that protects individuals from 'succumbing to adversity' (2003, p. 268). Such perceptions 'appear to reflect a pervasive worldview, rooted perhaps in childhood experiences and attachment history' while domain-specific social support is 'more clearly the result of experiences with particular relationships and tend to influence only judgments that are most closely tied to these relationships' (Davis et al. 1998, p. 478).

As a multidimensional construct, social support is often measured by the size of the social network, the quality and frequency of contact with members of the social network, as well as instrumental and emotional forms of support received (Barrera 1986; George 1989; Tardy 1988). Typical domains of social support reported in the research literature have included family members, friends and significant others.

Davis, Morris and Kraus (1998) assessed social support available to college students across four specific domains including family, friends, romantic partners and faculty advisors. They expected the number of potential figures within each specific domain to vary, but they were interested in perceptions of support from each domain and not the size of the support network. They found that respondents made 'fairly sharp distinctions' between different social domains and perceptions of global social support. Friends were identified as the strongest source of support followed by parents and romantic partner, while support from faculty advisors was only weakly associated with global support. Overall, the friends domain of social support accounted for the most powerful associations with wellbeing in their study. Their findings are consistent with Chou (2000), Clara et al. (2003), and Brock, Peirce and Sarason (1996), all of whom identified friends, parents and family as the most often reported social supports among high school and college students and also the domains of support most strongly associated with wellbeing.

It is important to note that global social support is not necessarily explained by the additive combination of domain-specific supports. One explanation for this is that the domain-specific support categories measured in previous research (i.e. parents, family, friends, significant other, faculty advisor) may have been too specific, and may not have included an adequate range of possible supporters to capture the full array of support perceived to be available (Davis et al. 1998). Thus while global and domain-specific social supports are associated with wellbeing outcomes, they are also distinct constructs (Davis et al. 1998). Consequently, future research should take a broader view of the measures of specific social support domains.

It is also important to address a wider array of psychological wellbeing measures that may be impacted by social support. Much of the research to date has examined depression and anxiety as indices of wellbeing but much less emphasis has been placed upon strengths-based indices of wellbeing such as satisfaction with life. Therefore, it would be valuable to understand the unique associations with positive measures of wellbeing that specific domains of social support, in particular 
perceived support from friends as well as from caring adults, may have in the lives of college students.

\section{Present investigation}

Of greatest interest to the present research was the broad domain of social support perceived to be available from caring and safe adults. To address the need for greater breadth in measurements of social support domains, I did not limit this domain by any specific category of adult. Instead, I simply asked respondents how many safe adults (not peers) they had to turn to in difficult times. I was primarily interested not only in the size of this domain-specific social support of primary interest, but also in the identification of who these safe adults were that college students felt they could turn to.

To address the need for a wider array of psychological wellbeing assessments as they relate to perceptions of social support, I included several strengths-based and positive wellbeing measures in this study. Both academic performance and satisfaction with life seemed to be key indicators of healthy and positive wellbeing outcomes among college students. Doing well academically and feeling satisfied with one's life would be for many a successful college experience. Additionally, positive wellbeing among college students is also thought to be indicated by higher levels of emotional intelligence (EI) as EI is assumed to reflect characteristics that enable a person to attend to and value feelings, while also being clear about the meaning of feelings and the expression of them (Gohm and Clore 2002). Therefore, higher levels of emotional intelligence might be argued to lead to higher levels of wellbeing and better everyday coping and problem solving (Gohm and Clore 2002), which may be associated with specific domains of perceived social support, in particular safe and caring adults.

Given that both stress (Misra and Castillo 2004) and problematic alcohol consumption patterns (Ham and Hope 2003; O’Malley and Johnston 2002) are prevalent and often perennial challenges faced by many college students as they transition to college life and face the academic pressures associated with it, it is valuable to examine both factors with regard to perceived social support domains. Perceived stress is highly correlated with depressive symptomatology and, although not a measure of depression, lower levels of perceived stress typically reflect higher levels of wellbeing and lower scores on depression measures.

Problematic alcohol consumption patterns among college students may be a means of coping with perceived stress and may also vary in relation to perceptions of social support available from specific support domains. Additionally, other coping strategies used by college students in dealing with their day-to-day challenges should be explored in relation to perceptions of social supports. It might be argued that perceptions of more caring and safe adults to turn to in difficult times may provide not only support but opportunities to develop better and healthier coping skills than might be developed when turning to one's peers for support.

In the present study, I intended the domain of safe adult social support to be broader and more inclusive than previous studies, while I intended the social support domain 
of 'friends' to be more specific with regard to the type of 'friend' associated with measures of wellbeing, specifically close versus casual. Therefore, I examined three domains of perceived social support: (1) the number of close friends reported, (2) the number of casual friends reported, and (3) the number of safe adults they felt safe turning to in difficult times (see Figure 1).

I examined the associations between these three domain-specific social supports in relation to three subjective wellbeing (SWB) areas including (1) negative SWB, (2) positive SWB, and (3) coping strategies (see Figure 1). Negative SWB measures included perceived stress and patterns of problem alcohol consumption (the CAGE, AUDIT and substance use subscale from the Brief Cope measure). Positive SWB measures included academic performance (e.g. self-reported GPA), satisfaction with life, overall emotional intelligence (EI), and four subscales of EI (self-emotion appraisal, other-emotion appraisal, regulation of emotion and use of emotion). Finally, I measured coping outcomes using the Brief Cope scale, resulting in four subscales: emotion-focused coping, problem-focused coping, other coping mechanism: adaptive, and other coping mechanism: maladaptive (see Figure 1).

I expected that, among the three specific domains of social support examined, having more safe adults to turn to in difficult times would yield the greatest associations with measures of wellbeing among college students than either having more close or casual friends would yield. Additionally, I expected that having more close friends would yield greater associations to measures of wellbeing than would having more casual friends. My expectations were based on previous findings indicating that perceived support from families (Chou 2000) and sometimes both families and friends (Clara et al. 2003) was associated with lower levels of depression, while at least one study (Chou 2000) found that support from friends was associated with lower levels of anxiety but not depression. Because relationships with 'close' friends and also 'safe' adults would suggest some level of trust and intimacy as part of the relationship, the same assumption would not necessarily be made within the relationships with 'casual' friends. Therefore, I expected to find fewer associations with wellbeing with the casual friends domain of social support as compared to the other two domains of support.

I also expected that social support from safe adults would yield the greatest number of associations with coping measures. In part, this was due to the assumption that college students who reported more safe adults to turn to in difficult times may actually have had encounters with these adults in the past that promoted the development of better coping strategies in dealing with life's challenges. Although close friends may provide resources of social support, they may also be at the same developmental stage in life, and therefore such support may yield fewer associations with effective coping strategies. 
Figure 1: Social support domains and measures of wellbeing

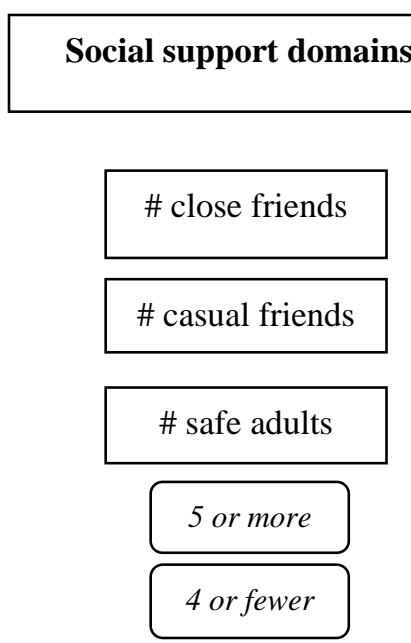

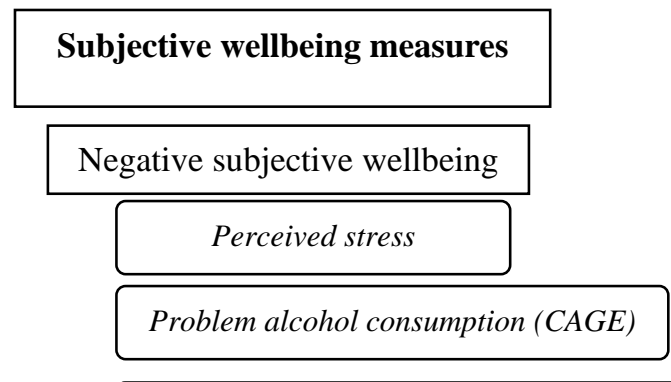

Hazardous alcohol consumption (AUDIT)

Alcohol dependence (AUDIT)

Harmful alcohol problems (AUDIT)

Substance use subscale (BCOPE)

Positive subjective wellbeing

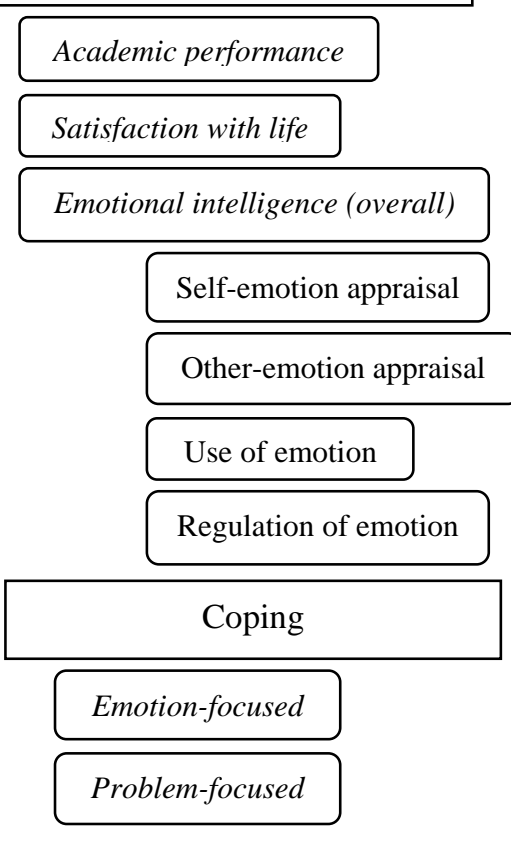

Other: adaptive

Other: maladaptive 
Finally, I wanted to examine more closely the patterns of associations with wellbeing of two levels of safe adults in the lives of college students. Specifically, I compared those with more versus fewer perceived safe adults to turn to in difficult times on all wellbeing measures. I expected that comparisons between those who reported four or fewer safe adults to turn to in difficult times as compared to those with five or more would result in weaker and fewer associations with wellbeing measures as well as differences in coping strategies.

\section{Method}

\section{Participants}

The overall sample included 259 respondents from a small, Catholic, residential college in the northeast region of the United States, comprised of 118 (46\%) males and 141 (54\%) females whose average age was 20 years. The majority of respondents were white (246) (95\%) and heterosexual (254) (98\%). A smaller sample of 75 respondents completed the Brief Cope outcome measures due to a change in the survey instrument. Of those respondents, 30 (40\%) were male, 43 (57\%) were female, and the average age of respondents was 19.

\section{Procedure}

The majority of participants (75\%) were approached at various locations around the college campus and asked if they would be willing to volunteer to participate in a survey on health and wellbeing among college students. Other participants (25\%) were volunteers from introductory and other psychology classes who received extra credit for their participation. The survey included measures of wellbeing related to this research study and required 30-40 minutes for completion. The three questions about domain-specific social supports were located in a lengthy demographic section at the end of the survey (i.e. 'How many close friends do you have?', 'How many casual friends do you have?' and 'How many safe adults (not your peers) do you have to turn to in difficult times?'). All participants read and signed an informed consent form and were also debriefed in writing following their participation. The debriefing form explained that several measures associated with wellbeing were included on the survey and that the research was interested in whether different domains of social support (close friends, casual friends or safe adults) might yield more beneficial outcomes to college students.

\section{Measures}

\section{Negative SWB measures}

I used the Perceived Stress Scale (PSS) (Cohen, Kamarck and Mermelstein 1983) as a brief 14-item measure of the degree to which situations in one's life are appraised as stressful. It is highly correlated with depression symptomatology measures and assesses states that place people at risk for clinical disorders. For example, '[I]n the last month, how often have you felt nervous and "stressed"?', and '[I]n the last month, how often have you felt that you were on top of things?' scores are summed and range from 14 to 56 . The mean score for college students ranges between 23 and 25, with a mean of 23.18 for females and 23.67 for males. 
I measured problem alcohol consumption patterns using three scales including the CAGE, AUDIT and the Substance Use scale of the Brief Cope measure. The CAGE is an assessment instrument used internationally for identifying those experiencing problems with alcohol consumption (Ewing 1984), which requires less than a minute to complete. CAGE is an acronym that stands for Cut-Annoyed-Guilty-Eye and corresponds to the four items on the questionnaire: (1) Have you felt the need to stop or cut down your drinking? (2) Have you been angry or annoyed at other people talking about or criticising your drinking? (3) Have you felt guilty as a result of something you did when you were drinking? (4) Have you ever taken a drink first thing in the morning? (eye-opener/early morning drinking). These very straightforward questions are responded to by 'yes' (score of 1) or 'no' (score of 0) and scores are derived by summing the responses from the four questions. A score of two indicates possible problematic drinking while a score of three or four indicates problematic drinking and potential dependence. The CAGE has been found to be better at identifying lifetime alcohol abuse and dependence while the Alcohol Use Disorders Identification Test (AUDIT) is used for the detection of hazardous and harmful drinking (McCusker et al. 2002).

The AUDIT was developed by the World Health Organization as a swift screening method for excessive drinking and is a sensitive questionnaire that detects hazardous and harmful drinking patterns (Saunders et al. 1993). It consists of 10 questions with response scales ranging from 0 to 4 , where 0 indicates 'never' and 4 may indicate 'daily or almost daily', resulting in an overall maximum score of 40. It assesses subscales of hazardous alcohol consumption (e.g. frequency of drinking, quantity and frequency of heavy drinking), alcohol dependence (e.g. impaired control over drinking, increased salience of drinking and morning sickness), and harmful alcohol problems (e.g. guilt after drinking, blackouts, alcohol-related injury and others concerned about drinking). A score between 0 and 7 would recommend alcohol education, between 8 and 15 would recommend simple advice, between 16 and 19 would recommend simple advice plus counselling and monitoring, and scores between 20 and 40 would recommend referral to a specialist for diagnostic evaluation and treatment.

The third measure of problem alcohol consumption was the Substance Use Subscale of the Brief COPE (Carver 1997). This subscale assesses the degree to which respondents turn to the use of alcohol or other drugs as a way of disengaging from the stressor. It includes two statements: 'I've been using alcohol or drugs to make myself feel better' and 'I've been using alcohol or drugs to help me get through it' Respondents offer responses on a scale from 1, 'I haven't been doing this at all' to 4, 'I've been doing this a lot'. A higher score indicates greater risk of alcohol abuse.

\section{Positive SWB measures}

Positive subjective wellbeing measures include academic performance, satisfaction with life and emotional intelligence. I assessed academic performance through selfreported cumulative GPA and also GPA within the respondent's major (ranging from 0 to 4.0). I assessed satisfaction with life using the satisfaction with life scale (Diener et al. 1985), designed to assess one's satisfaction with life as a whole. The measure is composed of five questions including: 'I consider my life close to ideal' 
and 'Overall I am satisfied with my life'. Each of the questions is scored on a 7point Likert Style scale ( $1=$ strongly disagree to $7=$ strongly agree). The overall score is a total of the five items, with scores ranging from 5 to 35, with 5 indicating not at all satisfied with life and 35 indicating total satisfaction with life. This measure has been shown to have high internal consistency and reliability and correlates well with other measures of subjective wellbeing (Diener et al. 1985).

I assessed emotional intelligence using Wong and Law's (2002) Emotional Intelligence Scale (EI), a 16-item self-report measure. An overall emotional intelligence scale score and four subscale scores result. The subscales are (1) SelfEmotional Appraisal (SEA) (i.e. 'I have a good understanding of my own emotions'), (2) Others' Emotional Appraisal (OEA) (i.e. 'I am a good observer of others' emotions'), (3) Use of Emotion (UOE) (i.e. 'I am able to control my temper and handle difficulties rationally'), and (4) Regulation of Emotion (ROE) (i.e. 'I always set goals for myself and then try my best to achieve them'). The participants were asked to indicate on a 7-point Likert style scale, where 7 indicated strongly agree and 1 indicated strongly disagree, how strongly they agreed or disagreed with each of the sixteen statements. A higher score reflected individuals with higher levels of emotional intelligence.

I used the Brief COPE scale (Carver 1997) to assess coping strategies. A 28-item self-report measure based upon concepts of coping from Lazarus and Folkman (1984), it assesses how respondents typically react to stressful events. It is an abbreviated scale from a full Cope measure and yields 14 subscales of two items each, using a scale where 1 indicates 'I haven't been doing this at all', and 4 indicates 'I've been doing this a lot'.

The combination of these 14 subscales results in four primary subscales of coping including (1) emotion-focused coping, (2) problem-focused coping, (3) other coping mechanism: adaptive, and (4) other coping mechanism: maladaptive. Emotionfocused coping consists of subscales including use of emotional support (e.g. getting sympathy or emotional support from someone), positive reframing (e.g. making the best of the situation by growing from it, or viewing it in a more favorable light), and religion (e.g. increased engagement in religious activities). Problem-focused coping consists of three subscales including active coping (e.g. taking action, exerting effort to remove or circumvent the stressor), planning (e.g. thinking about how to confront the stressor, planning one's active coping efforts), and use of instrumental support (e.g. seeking assistance, information or advice about what to do).

'Other coping mechanism: adaptive' consists of acceptance (e.g. accepting the fact that the stressful event has occurred and is real) and humour (e.g. making jokes about the stressor) while 'other coping mechanism: maladaptive' consists of 6 subscales. They include venting (e.g. an increased awareness of one's emotional distress, and a concomitant tendency to ventilate or discharge those feelings), behavioural disengagement (e.g. giving up, or withdrawing effort from, the attempt to attain the goal with which the stressor is interfering), mental disengagement (e.g. self-distraction), psychological disengagement from the goal with which the stressor is interfering (e.g. daydreaming, sleep or self-distraction), self-blame (e.g. criticising 
or blaming oneself for the stressor that has occurred), substance use (e.g. turning to the use of alcohol or other drugs as a way of disengaging from the stressor), and denial (e.g. an attempt to reject the reality of the stressful event).

\section{Results}

I conducted correlational analyses between the number of close friends, the number of casual friends and the number of safe adults reported and measures of negative SWB, positive SWB and coping. I also conducted one-way analyses of variance to examine differences between those who perceived four or fewer safe adults to turn to in difficult times versus those with five or more safe adults to turn to in difficult times on the three wellbeing outcome areas (negative SWB, positive SWB and coping).

\section{Correlation analyses}

Close friends. Results indicated that having more close friends was associated with higher levels of satisfaction with life $(r=.15, p=.01)$, lower levels of perceived stress ( $r=-.17, p=.01$ ), but higher AUDIT alcohol consumption scores $(r=.24, p=.01)$ (see Figure 2). Having more close friends was also associated with lower levels of emotion-focused coping ( $r=-.29, p=.05)$, and other coping: maladaptive $(r=-.27$, $p=.05$ ), but also lower levels of other coping: adaptive $(r=-.28, p=.05)$ (see Figure 2).

Casual friends. Having more casual friends was not associated with any positive measures of wellbeing but was positively correlated with problematic alcohol consumption (AUDIT) $(r=.34, p=.01)$ and possible alcohol dependence (AUDIT) $(r=.19, p=.01)$ (see Figure 2). These results suggest that greater numbers of reported casual friends in this college sample was associated with heavy and or high-risk alcohol consumption patterns but not with any positive indices of wellbeing.

Safe adults to turn to. As expected, the greatest number of associations with wellbeing were found for perceived safe adult social support rather than either close or casual friends domains. Overall, having more safe adults to turn to in difficult times was correlated with lower levels of perceived stress $(r=-.16, p=.01)$, and lower CAGE problem drinking scores $(r=-.13, p=.05)$, higher levels of satisfaction with life $(r=.24, p=.01)$, and negatively correlated with self-distraction coping $(r=-.25$, $p=.033$ ) (see Figure 2). Finally, having more caring/safe adults to turn to was correlated with higher emotional intelligence scores $(r=.15, p=.01)$ including two subscales of emotional intelligence, specifically self-emotion appraisal $(r=.19$, $p=.01)$ and regulation of emotion $(r=.13, p=.01)$ (see Figure 2). Contrary to expectations, no associations were found with any of the coping measures.

One-way analyses of variance. I conducted one-way analyses of variance between respondents with $0-4$ or 5 or more safe adults to turn to in difficult times on (1) negative wellbeing measures (perceived stress, the substance use coping subscale from the Brief Cope scale, the AUDIT alcohol consumption and alcohol dependence scores, and the CAGE problem drinking scale score), (2) positive wellbeing measures (e.g. satisfaction with life, emotional intelligence (EI), and EI subscales including self-emotion appraisal, other-emotion appraisal, regulation of emotion and 
use of emotion) and (3) on coping outcome measures (e.g. coping strategies based on the 14 subscales of the Brief Cope scale).

Figure 2: Social support domains and correlates of wellbeing $(\mathrm{N}=259)$
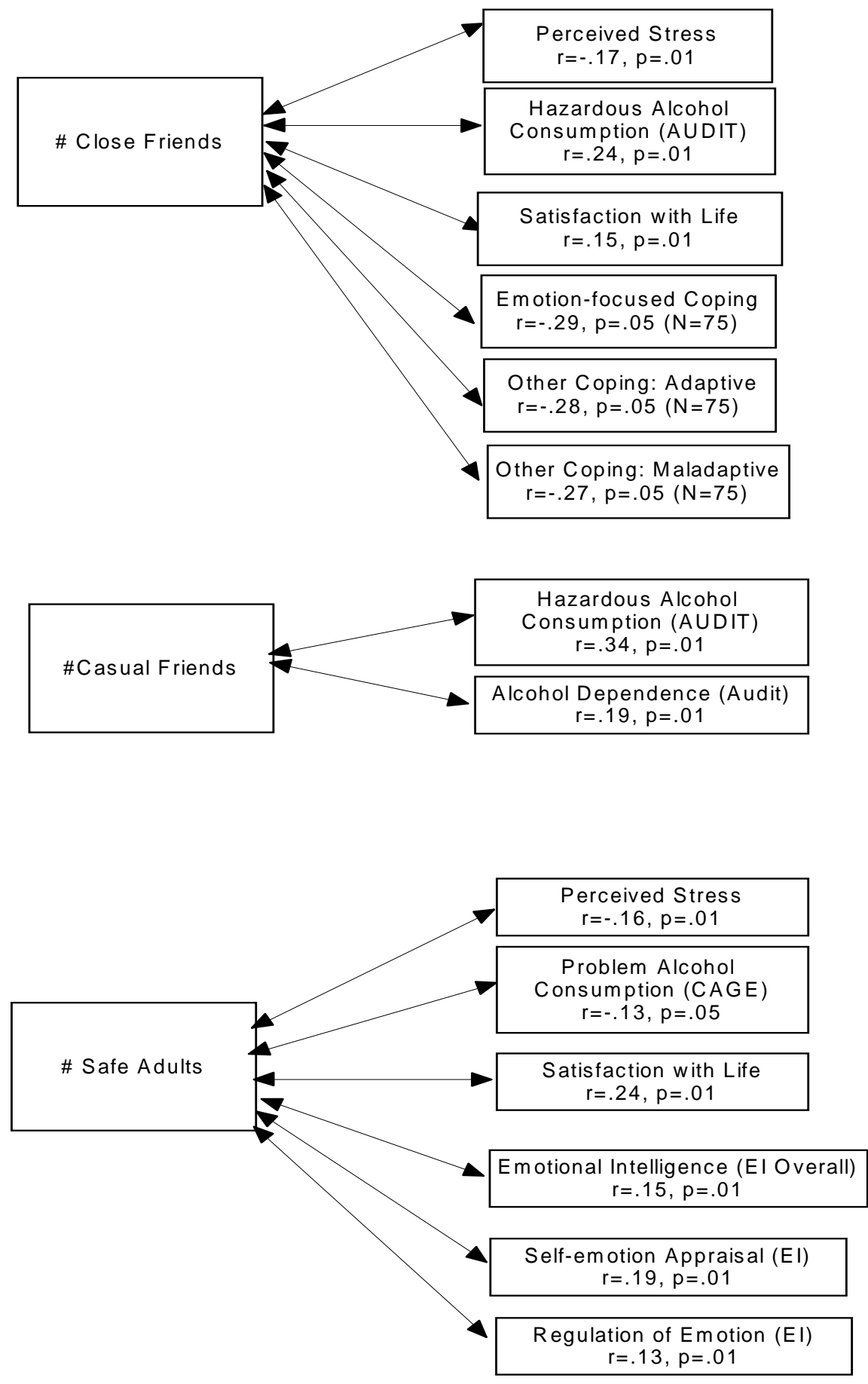
The two levels of safe adults were created by splitting the six response categories (i.e. $0,1-2,3-4,5-7,8-10,11+$ ) into two levels, specifically, 4 or fewer safe adults (i.e. $0,1-2,3-4)$ and 5 or more safe adults (e.g. 5-7, 8-10, 11+). Frequency distributions indicated that 56.8 per cent (147) of respondents comprised the 4 or fewer safe adults group and 43.2 per cent (112) of respondents comprised the 5 or more safe adults group.

Negative wellbeing. Results indicated perceived stress scores were significantly lower (and within the average range for college students) for respondents with 5 or more safe adults to turn to in difficult times $(M=24.1, s d=7.6)$ as compared to respondents with 4 or fewer safe adults to turn to $(M=26.6, s d=7.3)$ (above the average range) $(F(1,256)=7.49, p=.007, d=.34)$. However, no differences were found in alcohol problem indices including consumption (AUDIT), dependence (AUDIT), substance use as a coping method (BCOPE) or problematic drinking patterns (CAGE).

Positive wellbeing. Results from the examination of positive outcomes indicated that respondents with 5 or more caring/safe adults to turn to in difficult times reported significantly higher levels of satisfaction with life $(M=26.1, s d=4.8, M=24.0, s d=5.8$, respectively) $(F(1,255)=9.97, p=.002, d=.39)$, higher overall emotional intelligence scores $(M=86.3, s d=11.4, M=83.2$, $s d=12.2$, respectively $)(F(1,248)=4.21, p=.041$, $d=-.26)$, and higher scores on two emotional intelligence subscales, specifically selfemotion appraisal ( $M=23.0, s d=3.3, M=21.6, s d=3.6$, respectively) $(F(1,255)=11.0$, $p=.001, d=-.42)$ and regulation of emotion $(M=21.9, s d=4.3, M=20.8, s d=4.6$, respectively) $(F(1,255)=4.17, p=.042, d=-.26)$. In addition, respondents who had 5 or more caring/safe adults to turn to also performed better academically as indicated by both their self-reported cumulative GPA $(M=3.08, s d=.42, M=2.94, s d=.48$, respectively) $(F(1,214)=4.86, p=.029, d=.31)$ and GPA within their major $(M=3.24$, $s d=.47, M=3.05, s d=.52$, respectively $)(F(1,168)=5.5, p=.02, d=.38)$.

Coping measures. Coping strategies might be expected to differ between college students who perceived greater numbers of safe adults available to them and students who perceived fewer safe adults to turn to in difficult times. However, results yielded only one significant difference in coping strategies between these two groups. Specifically, respondents with five or more caring/safe adults to turn to reported lower scores on the self-blame coping measure (i.e. criticising and blaming oneself for the stressor) as compared to respondents with four or fewer caring/safe adults $(M=3.8, s d=1.5, M=4.6, s d=1.9$, respectively) $(F(1,69)=4.01, p=.049, d=-$ $.50)$.

Categories of adults to turn to in difficult times. Finally, I also assessed who the adults were that college students perceived they could turn to in difficult times to understand this broader social support domain more fully. Respondents were asked to check boxes on the survey of several categories of adults that constituted their estimated number of safe adults to turn to in difficult times including parents, relatives, teachers, religious leaders, neighbours, employers and other (followed by fill-in-the-blank). Response frequencies were examined between respondents who 
indicated five or more adults to turn to as compared to respondents with four or fewer caring/safe adults to turn to in difficult times.

The four most cited safe adult categories for the $5+$ and 4 or fewer safe adult levels were parents ( $94 \%$ and $80 \%$, respectively), relatives ( $85 \%$ and $60 \%$, respectively), teachers ( $55 \%$ and $27 \%$, respectively) and neighbours ( $41 \%$ and $18 \%$, respectively), followed by employers (33\% and $7.5 \%$, respectively), other (25\% and $16.3 \%$, respectively) and religious leaders (15\% and 5\%, respectively). Clearly, respondents with five or more caring/safe adults reported higher percentages of caring/safe adults in all of the categories. Nearly twice as many respondents with 5 or more caring/safe adults identified teachers and three times as many identified employers as caring/safe people to turn to than did respondents who perceived four or fewer caring/safe adults to turn to in difficult times.

\section{Discussion}

The transition to college can be a daunting experience and, even once there, navigating the many challenges of social life and academic expectations can continue to challenge even the best of students. For college students, the sense that they are loved, cared for and supported by friends, family members and others can make a significant difference in their overall wellbeing, especially in regard to depression and anxiety. But with growing understanding of the multidimensional nature of social support and its many correlates to wellbeing, published research has been criticised for limiting the way social support has been measured (Vaux, Riedel and Stewart 1987). Chou (2000) stated clearly that studies that fail to consider the source of social support may indeed lose very important information as to what and how these perceptions impact on wellbeing. Thus, in the present study, one domain of social support among college students designed to be broad was safe adults to turn to in difficult times. This domain-specific social support included any caring adults perceived to be safe to turn to in difficult times and did not draw distinctions between whether those adults were family, relatives, teachers, coaches, employers or others. In so doing, this domain was both broad and yet also distinct from peer friendships and romantic partner support domains.

Building upon the Davis et al. (1998) study, which assessed social support available to college students across four specific domains (family, friends, romantic partners and faculty advisors), the present research examined three specific domains of support: the number of close friends, casual friends and safe adults to turn to in difficult times. However, unlike the Davis et al. (1998) study, this study was indeed interested in the size of the domains of social support and not the perceptions of support provided by them. Therefore, instead of asking respondents which domains they perceived provided the most support and consequently contributed the most to wellbeing, this investigation measured several indices of wellbeing and coping which were then correlated individually with each of the three domain-specific social supports.

Two aspects of the present study differentiate it from other studies on social support, namely the safe adults to turn to domain of social support and the greater breadth of wellbeing measures included. First, the safe adults to turn to domain was broader 
than previous measures of adult social supports and was inclusive of any and all caring and safe adults that college students felt they had available for support during difficult times. Second, the measures of wellbeing in this study were also broad and included both deficit (e.g. perceived stress and problem alcohol consumption patterns) and strengths-based (satisfaction with life, emotional intelligence and academic performance) indices as well as measures of coping (four primary subscales of the Brief Cope scale).

I expected that the perception of more safe adults to turn to in difficult times among college students would be associated with more measures of wellbeing and would demonstrate more associations with wellbeing and coping than would either close or casual friends social support domains. I further expected that the casual friends domain of social support would yield the fewest associations to wellbeing of the three social support domains. Finally, I expected that comparisons between those who had four or fewer safe adults to turn to in difficult times as compared to those with five or more would result in weaker and fewer associations with wellbeing outcomes and differences in coping strategies as well.

The results were consistent with my expectations in that a greater number of associations with wellbeing measures were found for the safe adults domain of social support followed by the close and casual friends domains of social support. In fact, no indices of positive wellbeing were associated with the casual friends domain of social support at all. Overall, having more safe adults to turn to in difficult times among college students was associated with six positive wellbeing outcomes, namely lower perceived stress, greater satisfaction with life, higher overall emotional intelligence (EI), self-emotion appraisal (EI subscale), regulation of emotion (EI subscale) and lower problem drinking (CAGE) scores. Similarly, having more close friends was associated with two positive wellbeing outcomes lower perceived stress and greater satisfaction with life - but, unlike the safe adults domain, having more close friends was also associated with higher problem alcohol consumption (AUDIT) scores. Not only were close friends associated with alcohol consumption, but having more casual friends as a college student was associated with both higher problem alcohol consumption (AUDIT) and higher possible alcohol dependence (AUDIT) scores.

I also expected that coping strategies would be different between the domains of social support. I thought that better coping strategies would be associated with the domain of safe adult social support more than for close or casual friend supports, and indeed there were differences but none that reflected any significant benefits associated with safe adults as I expected. Respondents who indicated having more safe adults to turn to reported only lower mental disengagement (e.g. selfdistraction) coping strategies, while respondents who indicated having more close friends reported lower 'other: maladaptive' coping (e.g. venting, behavioural disengagement, mental disengagement, self-blame, substance use and denial), but also lower emotion-focused coping (e.g. emotional support and positive reframing) and lower 'other: adaptive' coping (e.g. acceptance and humour) strategies. 
Last, I expected that examination of the safe adults domain of social support would yield more associations with wellbeing for respondents who indicated that they had five or more safe adults to turn to as compared to those with four or fewer safe adults to turn to. Consistent with my expectations, college students with five or more safe adults to turn to in difficult times reported significantly lower levels of perceived stress, greater satisfaction with life, higher overall emotional intelligence (EI) scores, self-emotion appraisal (EI subscale), regulation of emotion (EI subscale), higher academic achievement (both cumulative and major GPAs), and also lower self-blame coping scores than respondents with four or fewer safe adults to turn to. Clearly, having a smaller network of these adults perceived to be safe, caring and available in trying times yielded significantly fewer associations with wellbeing among college students.

Consistent with previous research, the present findings suggest that certain domains of social support including safe adults to turn to and close friends are associated with positive wellbeing among college students and may be considered contributing factors to a buffering effect from adversity. While Davis et al. (1998) found that 'friends' accounted for the most powerful associations with wellbeing in comparison to family, romantic partners and faculty advisors, the present study found this to be true for only a certain type of 'friend', specifically close friends but not casual friends. Having more 'close friends' was associated with lower perceived stress and higher satisfaction with life scores but having more 'casual friends' was associated only with measures related to problematic alcohol consumption patterns. Even though having more close friends was associated with certain aspects of positive wellbeing, it too was associated with problem alcohol consumption patterns among college students. In fact, the only domain of social support in this study associated with lower problem drinking scores was that of safe adults to turn to. College students who perceived and reported more safe adults to turn to in difficult times tended to report lower CAGE problem drinking scores, suggesting lower levels of lifetime alcohol abuse and dependence.

The perception of having more safe adults to turn to in difficult times, in addition to being the only domain associated with lower problem drinking, was also the only domain of social support associated with overall emotional intelligence (EI) and two EI subscales: self-emotion appraisal and regulation of emotion. Emotional intelligence is assumed to be associated with an awareness and adeptness in dealing with one's own feelings as well as in reading the emotional states of those around us. Those higher in emotional intelligence are assumed to have higher levels of mental health and better coping and problem-solving skills and capacities (Gohm and Clore 2002).

Finally, not only is it important that we feel supported and cared for by those in our lives but how we cope with challenges also impacts on our wellbeing. Coping is generally understood as a process that involves appraisal of situations and the use of skills or strategies to manage or reduce the stressful circumstances (Lazarus 1966; Folkman and Lazarus 1980, 1985). Lazarus (1993) emphasised two styles of coping - problem-focused coping and emotion-focused coping - and although the two styles are viewed separately they may also occur congruently in dealing with a wide 
array of stressful situations. While problem-focused coping involves changing something in the situation or acting directly to remove the cause of stress, emotionfocused coping involves the reduction or management of the emotional distress associated with the situation (Sica et al. 1997).

The present research found that having more close friends was associated with lower emotion-focused coping, lower other: maladaptive coping, but also lower other: adaptive coping. It would appear that close friends may indeed contribute to a buffering effect from adversity, as Davis et al. (1998) found, where having more close friends was related to less need to regulate emotion with regard to situational distressors and was associated with lower levels of maladaptive coping. It may very well be that it is within the realm of coping where wellbeing is most greatly impacted by perceived social support from 'friends', as the Davis et al. (1998) study reported.

Although it might be expected that perceiving more connections with caring/safe adults might have resulted in greater contact with them and consequently more effective coping strategies, the results of this study did not support this. Instead, it would appear that college students cope very similarly regardless of whether they have more or fewer caring/safe adults in their lives to turn to in difficult times with the exception of mental disengagement (e.g. self-distraction) and self-blame (e.g. criticising and blaming oneself for the stressor). Young adults may not necessarily be learning coping skills to help them deal with stressors as a function of their relationships with caring/safe adults, but instead may simply be benefiting from feeling supported and cared for by these adults as they navigate certain stressful challenges of life.

It would appear from the findings of this study that both close friends and safe adults are valuable resources when it comes to coping with life's challenges. However, the two domains may function quite differently with regard to the type of support, the quality of support, and the possible timing with which college students seek support. Close friends possibly because of their proximity and availability may provide more immediate coping resources that reduce the need to seek out emotional support (i.e. sympathy) and positive reframing (i.e. making the best of the situation) as well as needs for venting and distraction or denial. Alternatively, having safe adults to turn to in difficult times may reduce the need to mentally disengage and distract oneself from the problem, possibly as a function of the perceived availability of a wiser adult who may provide support, guidance and reassurance that a peer may not be able to provide. Possibly the safe adults that college students turn to in difficult times are sought out when close friends are not able to provide the type or quality of support or assurance that life experience affords, whereas trustworthy and safe adults are able to assure them that everything will be alright. The data from this research did not find problem-focused coping occurred in association with having more safe adults to turn to and, thus, it may be that safe adults are not assisting in the promotion of problem-focused coping skills but may simply provide a different caring response to the stressful situation than close friends are able to offer. 
Perhaps the most important and interesting findings from this study were those related to college students who reported having four or fewer safe adults to turn to in difficult times as compared to those who reported five or more safe adults to turn to in difficult times. Those who perceived that they had five or more safe adults to turn to in difficult times reported lower perceived stress scores, higher satisfaction with life scores, higher overall emotional intelligence scores, higher self-emotion appraisal, higher regulation of emotion, higher self-reported academic performance (both cumulative GPA and term GPA), and lower levels of self-blame coping than college students with four or fewer safe adults to turn to in difficult times.

In conclusion, although I measured three domains of support networks in this study, namely the number of close friends, casual friends, and safe adults to turn to, in the present research I was most interested in the number of safe adult supports perceived to be available to college students in difficult times. Although much more research is needed, these results suggest that a valuable and simple screening assessment might assist college personnel to identify students at greater risk of 'succumbing to adversity' and who may be at risk of lower levels of wellbeing while at college. Could it be as simple as asking students to report the number of safe adults they perceive to be available to them in difficult times? For respondents in the present study who indicated they had four or fewer safe adults to turn to, it would seem to be the case.

Although much more research is needed, screening incoming students with a simple question about how many safe adults they have to turn to in difficult times might in fact provide a pathway for administrators and faculty to assist incoming first year students (as well as returning students) in developing connections with safe and caring adults at the start of their college career. Such a process may increase the likelihood of certain students having more successful academic and social experiences during one of the most important developmental periods of their life.

Future research should continue to examine the role of close friends and caring, safe adults as social supports in the lives of college students given the findings from the present study. It should also incorporate many more measures of positive wellbeing associated with these and other domains of support in order to understand better the breadth of positive impact that close and caring adults can have on adolescents and young adults today. Finally, research should also examine the qualitative differences in perceptions of social support available from different domains including who supporters are defined as and what characterises the type and value of support provided. 


\section{References}

Barrera, M 1986, 'Distinctions between social support concepts, measures, and models’, American Journal of Community Psychology, vol. 14, pp. 413-445.

Brock, DM, Pierce, GR, Sarason, BR 1996, 'Simultaneous assessment of perceived global and relationship-specific support', Journal of Social and Personal Relationships, vol. 13, pp. 143-152.

Carver, CS 1997, 'You want to measure coping but your protocol's too long: consider the Brief Cope', International Journal of Behavioral Medicine, vol. 4, no. 1, pp. 92-100.

Chou, K-L 2000, 'Assessing Chinese adolescents' social support: the multidimensional scale of perceived social support', Personality and Individual Differences, vol. 28, pp. 299-307.

Clara, I, Cox, B, Enns, M, Murray, L and Torgudc, C 2003, 'Confirmation factor analysis of the multidimensional scale of perceived social support in clinically distressed and student samples', Journal of Personality and Assessment, vol. 81, pp. 265-270.

Cohen, S, Kamarck, T and Mermelstein, R 1983, 'A global measure of perceived stress’, Journal of Health and Social Behavior, vol. 24, pp. 385-396.

Davis, MH, Morris, MM and Kraus, LA 1998, 'Relationship-specific and global perceptions of social support: associations to well-being and attachment', Journal of Personality and Social Psychology, vol. 74, no. 2, pp. 468-481.

Diener, E, Emmons, R, Larsen, R and Griffin, S 1985, 'The satisfaction with life scale’, Journal of Personality and Assessment, vol. 49, pp. 71-75.

Ewing, JA 1984, 'Electric aversion and individualized imagery therapy in alcoholism: a controlled experiment’, Alcohol, vol. 1, no. 2, pp. 101-104.

Folkman, S and Lazarus, RS 1980, 'An analysis of coping in a middle-aged community sample’, Journal of Health and Social Behavior, vol. 21, pp. 219-239.

Folkman, S and Lazarus, RS 1985, 'If it changes it must be a process: a study of emotion and coping during three stages of a college examination', Journal of Personality and Social Psychology, vol. 48, pp. 150-170.

George, L 1989, 'Social and economic factors' in Geriatric psychiatry, ed. EW Buse and DG Blazer, American Psychiatric Press, Washington, DC, pp. 203-234.

Gohm, CL and Clore, GL 2002, 'Four latent traits of emotional experiences and their involvement in well-being, coping, and attributional style', Cognition and Emotion, vol. 16, no. 4, pp. 495-518. 
Ham, LS and Hope, DA 2003, 'College students and problematic drinking: a review of the literature’, Clinical Psychology Review, vol. 23, pp. 719-759.

Lazarus, RS 1966, 'The role of coping in the emotions and how coping changes over the life course' in Handbook of emotion, adult development, and aging, ed. C Magai and SH McFadden, Academic Press, San Diego, CA, pp. 289-306.

Lazarus, RS and Folkman, S 1994, Stress, appraisal, and coping, Springer, New York.

Lazarus, RS 1993, 'Coping theory and research: past, present, and future', Psychosomatic Medicine, vol. 55, pp. 234-247.

McCusker, MT, Baquille, J, Khwaja, M, Murray-Lyon, IM and Catalan, J 2002, 'Hazardous and harmful drinking: a comparison of the AUDIT and CAGE screening questionnaires', QJM: Monthly Journal of the Association of Physicians, vol. 95, no. 9, pp. 591-595.

Misra, R and Castillo, LG 2004, 'Academic stress among college students: comparison of American and international students', International Journal of Stress Management, vol. 11, pp. 132-148.

O’Malley, PM and Johnston, LD 2002, 'Epidemiology of alcohol and other drug use among American college students', Journal of Studies on Alcohol, no. 14, pp. 23-39.

Sarason, IG, Sarason, BR and Pierce, GR 1990 'Social support: the search for theory’, Journal of Social and Clinical Psychology, vol. 9, pp. 133-147.

Saunders, JB, Aasland, OG, Babon, TF, De Le Fuente, JR and Grant, M 1993, 'Development of the Alcohol Use Disorders Identification Test (AUDIT): WHO collaborative project on early detection of persons with harmful alcohol consumption: II', Addiction, vol. 88, pp. 791-804.

Sica, C, Novara, C, Dorz, S and Sanovio, E 1997, 'Coping strategies: evidence of cross-cultural differences? A preliminary study with the Italian version of coping orientations to problems experienced (COPE)', Personality and Individual Differences, vol. 23, no. 6, pp. 1025-1029.

Tardy, CH 1988, 'Social support measurement', American Journal of Community Psychology, vol. 13, pp. 187-202.

Vaux, A, Riedel, S and Stewart, D 1987, 'Modes of social support: the Social Support Behaviors (SS-B) Scale’, American Journal of Community Psychology, vol. 15, pp. 209-237. 
Wong, CS and Law, KS 2002, 'The effect of leader and follower emotional intelligence on performance and attitude: an exploratory study', Leadership Quarterly, vol. 13, pp. 243-274. 